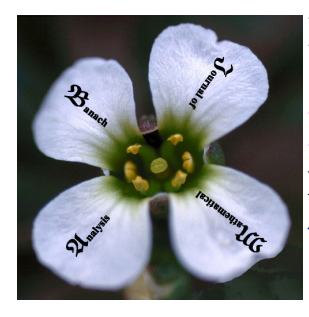

Banach J. Math. Anal. 8 (2014), no. 2, 204-213

B anach $\mathbf{J}_{\text {ournal of }} \mathbf{M}_{\text {athematical }} \mathbf{A}_{\text {nalysis }}$

ISSN: $1735-8787$ (electronic)

www.emis.de/journals/BJMA/

\title{
ON THE CONTINUITY OF THE GROUP INVERSE IN $C^{*}$-ALGEBRAS
}

\author{
J. BENÍTEZ ${ }^{1 *}$, D. CVETKOVIĆ-ILIĆ ${ }^{2}$ AND X. LIU \\ Communicated by D. Bakić
}

\begin{abstract}
Let $\left\{a_{n}\right\}_{n=1}^{\infty}$ be a sequence of group invertible elements of a unital $C^{*}$ algebra $\mathcal{A}$ that converges to $a$. We present some equivalent conditions for the group invertibility of $a$ and for the convergence of $\left\{a_{n}^{\#}\right\}_{n=1}^{\infty}$ to $a^{\#}$.
\end{abstract}

\section{INTRODUCTION}

Let $\mathcal{A}$ be a $C^{*}$-algebra. An element $a \in \mathcal{A}$ is Drazin invertible, if there exists $x \in \mathcal{A}$ such that

$$
a x=x a, \quad x a x=x, \quad a x a-a \text { is nilpotent. }
$$

Such $x$, if it exists, is unique and it is called the Drazin inverse of $a$ and is denoted by $a^{\mathrm{D}}$. The index of nilpotency of $a x a-a$ is denoted by $i(a)$. If $i(a)=k$, then Drazin inverse of $a$ satisfies

$$
a^{\mathrm{D}} a a^{\mathrm{D}}=a^{\mathrm{D}}, \quad a a^{\mathrm{D}}=a^{\mathrm{D}} a, \quad a^{k+1} a^{\mathrm{D}}=a^{k},
$$

and $k$ is the smallest integer such that (1.1) is satisfied. In the special case when $i(a)=1$, the Drazin inverse $a^{\mathrm{D}}$ is known as the group inverse of $a$, and it is denoted by $a^{\#}$. An element $a$ is invertible if and only if $i(a)=0$ and in this case $a^{\mathrm{D}}=a^{-1}$. The subset of group invertible and invertible elements of $\mathcal{A}$ is denoted by $\mathcal{A}^{\#}$ and $\mathcal{A}^{-1}$, respectively.

Date: Received: Nov. 11, 2013; Accepted: Dec. 29, 2013.

* Corresponding author.

2010 Mathematics Subject Classification. Primary 46L05; Secondary 46K05.

Key words and phrases. Continuity, group inverse, $C^{*}$-algebras, generalized inverses. 
Another well known kind of generalized inverse is the Moore-Penrose inverse. If for $a \in \mathcal{A}$, there exists $x \in \mathcal{A}$ such that

$$
a x a=a, \quad x a x=x, \quad(a x)^{*}=a x, \quad(x a)^{*}=x a,
$$

then we say that $a$ is Moore-Penrose invertible. Such $x$ is denoted by $a^{\dagger}$ and called the Moore-Penrose inverse of $a$. By $\mathcal{A}^{\dagger}$, we denote the subset of $\mathcal{A}$ consisting of all Moore-Penrose invertible elements.

An element $a$ in a $C^{*}$-algebra $\mathcal{A}$ is said to be regular when $a \in a \mathcal{A} a$. A basic result in the theory of generalized inverses is that any regular element in a $C^{*}$-algebra is Moore-Penrose invertible.

Contrary to the usual inverse of a square matrix, it is well-known that the Drazin inverse and the Moore-Penrose generalized inverse of a matrix are not necessarily a continuous function of the elements of the matrix. For the first time, the continuity of the Drazin inverse on the set of matrices was studied by S.L. Campbell and C.D. Meyer [5]. They proved that for the set of convergent matrices $\left(A_{n}\right)_{n \in \mathbb{N}}$ that converges to the matrix $A$,

$$
A_{n}^{\mathrm{D}} \rightarrow A^{\mathrm{D}} \Leftrightarrow\left(\exists n_{0} \in \mathbb{N}\right)\left(\forall n \geq n_{0}\right) \quad r\left(C_{n}\right)=r(C),
$$

where $A_{n}=C_{n}+N_{n}$ and $A=C+N$ are the core nilpotent decompositions of $A_{n}$ and $A$, respectively and $r(C)$ is the rank of the matrix $C$.

The continuity of the Drazin inverse on the set of bounded operators on Banach spaces, was considered by V. Rakočević [18], and later by V. Rakočević and J.J. Koliha [12] for the Banach algebra setting. See also [9].

Theorem 1.1. [12] Let $a_{n}$ and a be Drazin invertible elements of the Banach algebra $\mathcal{A}$ such that $a_{n} \rightarrow a$. Then

$$
a_{n}^{\mathrm{D}} \rightarrow a^{\mathrm{D}} \Longleftrightarrow \sup _{n}\left\|a_{n}^{\mathrm{D}}\right\|<\infty \Longleftrightarrow a_{n}^{\mathrm{D}} a_{n} \rightarrow a^{\mathrm{D}} a .
$$

Obviously, the case when $a_{n}$ and $a$ are group invertible elements follows directly by Theorem 1.1. But what happens if we do not suppose that $a$ is group invertible?

If we suppose that $\left(a_{n}\right)_{n \in \mathbb{N}}$ is a convergent sequence of group invertible elements from a $C^{*}$-algebra $\mathcal{A}$ such that $\left\{a_{n}\right\}_{n=1}^{\infty}$ converges to $a \in \mathcal{A}$, one of the problems studied in this paper is to characterize the case when $a \in \mathcal{A}^{\#}$.

If we assume that $a$ is Drazin invertible, by Theorem 1.1, we have that

$$
a_{n}^{\#} \rightarrow a^{\mathrm{D}} \Longleftrightarrow \sup _{n}\left\|a_{n}^{\#}\right\|<\infty \Longleftrightarrow a_{n}^{\#} a_{n} \rightarrow a^{\mathrm{D}} a \text {. }
$$

Since $a_{n} a_{n}^{\#} a_{n}=a_{n}$, for any $n \in \mathbb{N}$, by letting $n \rightarrow \infty$ we get $a a^{\mathrm{D}} a=a$, which means that $a$ is group invertible. But recall that we have assumed that $a$ is Drazin invertible (see [6]).

In this paper, we will present some equivalent conditions for the group invertibility of an element $a$ and for the continuity of group inverse in the case when $a$ is the limit of the convergent sequence $\left(a_{n}\right)_{n \in \mathbb{N}}$ of the group invertible elements from a $C^{*}$-algebra.

Beside the continuity of the Drazin inverse, the continuity of the Moore-Penrose inverse was studied by many researchers. Similarly, as in the matrix case for the 
Drazin inverse, G.W. Stewart [20] proved that for the convergent sequence of matrices $\left(A_{n}\right)_{n \in \mathbb{N}}$ that converges to the matrix $A$,

$$
A_{n}^{\dagger} \rightarrow A^{\dagger} \Leftrightarrow\left(\exists n_{0} \in \mathbb{N}\right)\left(\forall n \geq n_{0}\right) \quad r\left(A_{n}\right)=r(A) .
$$

The continuity of the Moore-Penrose inverse of an operator on Hilbert spaces has been studied by Izumino [11] under the additional assumption that $A_{n}, A$ are Moore-Penrose invertible. The analogous result is obtained in the $C^{*}$-algebra case in [17, Theorem 2.2], [8, Theorem 6], [19, Theorem 2.2]:

Theorem 1.2. Let $\mathcal{A}$ be a $C^{*}$-algebra and let $a_{n}, a \in \mathcal{A}^{\dagger}$ such that $a_{n} \rightarrow a$. Then

$$
a_{n}^{\dagger} \rightarrow a^{\dagger} \Longleftrightarrow \sup _{n}\left\|a_{n}^{\dagger}\right\|<\infty \Longleftrightarrow a_{n}^{\dagger} a_{n} \rightarrow a^{\dagger} a \Longleftrightarrow a_{n} a_{n}^{\dagger} \rightarrow a a^{\dagger} .
$$

Throughout this article we will assume that the $C^{*}$-algebra $\mathcal{A}$ has a unit $\mathbb{1}$.

\section{Results}

In this section, the word projection will be reserved for an element $p$ in a $C^{*}$ algebra which is idempotent $\left(p^{2}=p\right)$ and self-adjoint $\left(p^{*}=p\right)$. Evidently, if $p$ is a nonzero projection, then $\|p\|=1$. For a projection $p, \mathbb{1}-p$ is also a projection which will be denoted by $\bar{p}$.

For an element $a$ of a $C^{*}$-algebra $\mathcal{A}$, we denote by $\sigma(a)$ the spectrum of $a$ and by $r(a)$ the spectral radius of $a$. It is well known that $\sigma(a)$ is a nonempty compact subset of $\mathbb{C}$; if $\lambda \in \sigma(a)$, then $|\lambda| \leq\|a\|$; if $a$ is normal (i.e., $a a^{*}=a^{*} a$ ), then $r(a)=\|a\|$; if $a$ is self-adjoint (i.e., $a=a^{*}$ ), then $\sigma(a) \subset \mathbb{R}$.

Inspired by [10], in [1], the following defintion for the $C^{*}$-algebra case, is given:

Definition 2.1. Let $p, q$ be two projections in a $C^{*}$-algebra $\mathcal{A}$. The minimal angle between $p$ and $q$ is the number $\theta(p, q) \in[0, \pi / 2]$ such that $\cos \theta(p, q)=\|p q\|$.

Evidently, $\theta(p, q)=\theta(q, p)$. In the following theorem, we will prove some basic properties of $\theta(p, q)$. One can see [14, Theorem 3.1] for a related result. Let us remark that some other results concerning angles are proved, see e.g. [3, 4, 15]; but all these results are established -and proved- in a Hilbert space setting.

Theorem 2.2. Let $p, q$ be projections in a $C^{*}$-algebra $\mathcal{A}$ such that $p-q \in \mathcal{A}^{-1}$. Then

(i) $\left\|(p-q)^{-1}\right\|=\frac{1}{\sqrt{1-\|p q\|^{2}}}$ and $\left\|(p-q)^{-1}\right\|=1 / \sin \theta(p, q)$,

(ii) $\|p q\|=\|\bar{p} \bar{q}\|$ and $\cos \theta(p, q)=\cos \theta(\bar{p}, \bar{q})$.

Proof. Without loss of generality we can assume that $p, q$ are nontrivial projections. By Lemma 2.4 [14], we have that for $\lambda \in \mathbb{C} \backslash\{0,1,-1\}$,

$$
\lambda \in \sigma(p-q) \Longleftrightarrow 1-\lambda^{2} \in \sigma(p q) \text {. }
$$

Hence

$$
\begin{aligned}
\left\|(p-q)^{-1}\right\| & =r\left((p-q)^{-1}\right) \\
& =\frac{1}{\inf \{|\lambda|: \lambda \in \sigma(p-q)\}}=\frac{1}{\inf \left\{|\lambda|: \lambda^{2}=1-t, t \in \sigma(p q)\right\}} .
\end{aligned}
$$

Since by Lemma $2.4[14]: r(p q)=\|p q\|^{2}$ and $\sigma(p q) \subset[0,1]$, the item $(i)$ is proved. 
To prove (ii), it is enough to observe that $\bar{p}-\bar{q}=q-p$ and to apply item (i) for the projections $\bar{p}$ and $\bar{q}$.

To motivate the following concept, let us recall (see Theorem 16 of [7]) that if $P$ and $Q$ are two $n \times n$ complex Hermitian and idempotent matrices, then there exists a unitary matrix $U$ such that

$$
P=U \operatorname{diag}\left(\left[\begin{array}{cc}
I_{p} & 0 \\
0 & 0
\end{array}\right], I_{q}, I_{r}, 0,0\right) U^{*}
$$

and

$$
Q=U \operatorname{diag}\left(\left[\begin{array}{cc}
C^{2} & C S \\
C S & S^{2}
\end{array}\right], I_{q}, 0, I_{s}, 0\right) U^{*}
$$

where $C=\operatorname{diag}\left(\cos \theta_{1}, \ldots, \cos \theta_{p}\right), S=\operatorname{diag}\left(\sin \theta_{1}, \ldots, \sin \theta_{p}\right), \theta_{1}, \ldots, \theta_{p} \in(0, \pi / 2)$ are the principal angles between the range spaces of $P$ and $Q, 0$ (repeated $q$ times) and $\pi / 2$ (repeated $\max \{r, s\}$ times). Since

$$
\begin{gathered}
P-Q=U \operatorname{diag}\left(\left[\begin{array}{cc}
S^{2} & -C S \\
-C S & -S^{2}
\end{array}\right], 0, I_{r},-I_{s}, 0\right) U^{*}, \\
{\left[\begin{array}{cc}
S^{2} & -C S \\
-C S & -S^{2}
\end{array}\right]=\left[\begin{array}{cc}
0 & -S \\
-S & 0
\end{array}\right]\left[\begin{array}{cc}
C & S \\
-S & C
\end{array}\right],}
\end{gathered}
$$

and $\left[\begin{array}{cc}C & S \\ -S & C\end{array}\right]$ is unitary, we get that

$$
\|P-Q\|= \begin{cases}1 & \text { if } \max \{r, s\} \neq 0 \\ \max \left\{\sin \theta_{i}: 0 \leq i \leq p\right\} & \text { if } \max \{r, s\}=0, p \neq 0 \\ 0 & \text { if } \max \{r, s\}=p=0\end{cases}
$$

Hence,

$$
\begin{aligned}
\| P & -Q \| \\
& =\max \{\sin \theta: \theta \text { is a principal angle between the ranges spaces of } P \text { and } Q\} .
\end{aligned}
$$

So, in the $C^{*}$-algebra settings, we introduce the following definition:

Definition 2.3. Let $p$ and $q$ be projections in a $C^{*}$-algebra $\mathcal{A}$. The maximal angle between $p$ and $q$ is the number $\psi(p, q) \in[0, \pi / 2]$ such that $\|p-q\|=\sin \psi(p, q)$.

This definition is correct, since by Akhiezer-Glazman equality (see e.g., [16, Lemma 1 (i)] for a $C^{*}$-algebra case): If $p$ and $q$ are projections in a $C^{*}$-algebra, then

$$
\|p-q\|=\max \{\|p \bar{q}\|,\|q \bar{p}\|\} .
$$

So, $\|p-q\| \leq 1$. Observe that from the Akhiezer-Glazman equality we have,

$$
\|p+q-\mathbb{1}\|=\|p-\bar{q}\|=\max \{\|p q\|,\|\bar{q} \bar{p}\|\}=\max \{\cos \theta(p, q), \cos \theta(\bar{p}, \bar{q})\},
$$

which in view of Theorem 2.2, under the additional hypothesis of the invertibility of $p-q$, implies that $\|p+q-\mathbb{1}\|=\cos \theta(p, q)$. It is interesting to compare this last equality with the equality of the third item of the next theorem.

The following result will be useful to express the main result in a more geometrical way. 
Theorem 2.4. Let $\mathcal{A}$ be a $C^{*}$-algebra and let $p, q \in \mathcal{A}$ be non trivial projections such that $p+q-\mathbb{1} \in \mathcal{A}^{-1}$. Then

(i) $\|p \bar{q}\|=\|q \bar{p}\|$ and $\theta(p, \bar{q})=\theta(\bar{p}, q)$.

(ii) $\sin \psi(p, q)=\cos \theta(p, \bar{q})$.

(iii) $\left\|(p+q-\mathbb{1})^{-1}\right\|=1 / \cos \psi(p, q)$.

(iv) $\theta(p, q) \leq \psi(p, q)$.

(v) If $p \neq q$, then $\theta(p, q)=\psi(p, q) \Longleftrightarrow \sigma(p q) \cap(0,1)=\left\{\|p q\|^{2}\right\}$.

Proof. (i) Since $p-\bar{q}=p+q-\mathbb{1} \in \mathcal{A}^{-1}$, by item (ii) of Theorem 2.2 we get $\|p \bar{q}\|=\|\bar{p} q\|$, so $\|p \bar{q}\|=\|q \bar{p}\|$.

(ii) It follows by the Akhiezer-Glazman equality and item (i).

(iii) By (i) of Theorem 2.2.

(iv) By $p(p+q-\mathbb{1})=p q$ and item (iii), we get

$$
1=\|p\|=\left\|p q(p+q-\mathbb{1})^{-1}\right\| \leq\|p q\|\left\|(p+q-\mathbb{1})^{-1}\right\|=\frac{\cos \theta(p, q)}{\cos \psi(p, q)} .
$$

It is enough to recall that $\cos :[0, \pi / 2] \rightarrow \mathbb{R}$ is a decreasing function.

(v) Let us remark that $\theta(p, q)=\psi(p, q) \Longleftrightarrow\|p-q\|^{2}+\|p q\|^{2}=1$.

$(\mathrm{v} \Rightarrow)$ By Lemma 2.4 (i,ii) of [14] we have that $\|p q\|^{2} \in \sigma(p q) \subset[0,1]$. If $p q=0$, since there exists $x \in \mathcal{A}$ such that $x(p+q-\mathbb{1})=\mathbb{1}$ after multiplying by $q$, we get $0=q$, which is impossible since $q$ is non trivial projection. If $\|p q\|=1$, then $\|p-q\|^{2}=1-\|p q\|^{2}=0$, which is impossible since $p \neq q$. Hence $\|p q\|^{2} \in \sigma(p q) \cap(0,1)$. If there exists $c \in(0,1) \cap \sigma(p q)$ such that $c \neq\|p q\|^{2}$, by Lemma 2.4 (ii) of [14] we have

$$
\|p q\|^{2}=r(p q)=\sup \{|\lambda|: \lambda \in \sigma(p q)\} \geq c .
$$

Recall that we have supposed $c \neq\|p q\|^{2}$. Thus, $\|p q\|^{2}>c$. Hence

$$
\|p-q\|^{2}=1-\|p q\|^{2}<1-c .
$$

Since $\sqrt{1-c} \notin\{0,1,-1\}$ and $c \in \sigma(p q)$, by Lemma $2.4(\mathrm{v})$ of [14] we get $\sqrt{1-c} \in$ $\sigma(p-q)$. But, in this case

$$
\|p-q\|=r(p-q)=\sup \{|\lambda|: \lambda \in \sigma(p-q)\} \geq \sqrt{1-c},
$$

which is a contradiction with (2.2).

$(\mathrm{v} \Leftarrow)$ Note that by $[14$, Lemma $2.4(\mathrm{i})]$ we have $\sigma(p q) \subset[0,1]$. Let $x=$ $p q+q p-p-q+\mathbb{1}$. From

$x p=p x, \quad x q=q x, \quad(p+q-\mathbb{1})^{2}=(\mathbb{1}-p+q)(p-q+\mathbb{1})=(p-q+\mathbb{1})(\mathbb{1}-p+q)$,

we get $p+q-\mathbb{1} \in \mathcal{A}^{-1}$ if and only if $p-q-\mathbb{1}, p-q+\mathbb{1} \in \mathcal{A}^{-1}$. Hence we can deduce that $\pm 1 \notin \sigma(p-q)$. Also observe that it is not possible that $\sigma(p-q)=\{0\}$ : if otherwise, since $p-q$ is normal, then $0=r(p-q)=\|p-q\|$, thus $p=q$ and by hypothesis, $\sigma(p q) \cap(0,1)=\left\{\|p q\|^{2}\right\}=1$, which is a contradiction. Since $p-q$ is self-adjoint, using Lemma 2.4 of [14], and Akhiezer-Glazman equality, we have 


$$
\begin{aligned}
& \|p-q\|=r(p-q)=\sup \{|\lambda|: \lambda \in \sigma(p-q)\} \\
& \quad=\sup \{|\lambda|: \lambda \in \sigma(p-q) \backslash\{0,1,-1\}\}=\sup \left\{|\lambda|: 1-\lambda^{2} \in \sigma(p q), \lambda \in(0,1)\right\} \\
& \quad=\sup \{\sqrt{1-\xi}: \xi \in \sigma(p q) \backslash\{0,1\}\} .
\end{aligned}
$$

Since $\sigma(p q) \subset[0,1]$, then $\sigma(p q) \backslash\{0,1\}=\sigma(p q) \cap(0,1)$. By hypothesis we have that $\sigma(p q) \cap(0,1)=\left\{\|p q\|^{2}\right\}$. Hence (2.3) implies that $\|p-q\|=\sqrt{1-\|p q\|^{2}}$.

Here, we shall characterize when the limit of a sequence composed of group invertible elements of a $C^{*}$-algebra $\mathcal{A}$ is also group invertible. Further Theorem 2.5 will be useful to establish this characterization. Observe that if $a \in \mathcal{A}^{\#}$, then $a a^{\#}$ is an idempotent and the norm of a nonzero idempotent is not lesser than 1.

Theorem 2.5. Let $\mathcal{A}$ be a $C^{*}$-algebra. If $a \in \mathcal{A}^{\#} \backslash\{0\}$, then

$$
\left\|a a^{\#}\right\|=\frac{1}{\cos \psi\left(a a^{\dagger}, a^{\dagger} a\right)} \text {. }
$$

Proof. It follows from Theorem 2.4 (iii), [2, Cor. 2.1 (i)], and [13, Prop. 4.2].

Next result gives an expression of $a^{\#}$ in terms of the Moore-Penrose inverse for an element $a \in \mathcal{A}^{\#}$. It is noteworthy that this result holds if we exchange a $C^{*}$-algebra by a unitary ring with an involution.

Theorem 2.6. Let $\mathcal{A}$ be a $C^{*}$-algebra. If $a \in \mathcal{A}^{\#}$, then

$$
\left(a^{\#}\right)^{\dagger}=a^{\dagger} a^{3} a^{\dagger} \text {. }
$$

Proof. Denote $p=a a^{\dagger}$ and $q=a^{\dagger} a$. By Theorem 2.3 and Corollary 2.1 of [2] we have

$$
p+q-\mathbb{1} \in \mathcal{A}^{-1} \quad \text { and } \quad a^{\#}=(p+q-\mathbb{1})^{-1} a^{\dagger}(p+q-\mathbb{1})^{-1} .
$$

From the equalities $(p+q-\mathbb{1}) a=q a$ and $a(p+q-\mathbb{1})=a p$ we have

$$
a=(p+q-\mathbb{1})^{-1} q a \quad \text { and } \quad a=a p(p+q-\mathbb{1})^{-1} .
$$

To prove the theorem, we will check that $(q a p)^{\dagger}=a^{\#}$ by the definition of the Moore-Penrose inverse.

$$
\begin{aligned}
&(q a p) a^{\#}(q a p)=q\left(a p(p+q-\mathbb{1})^{-1}\right) a^{\dagger}\left((p+q-\mathbb{1})^{-1} q a\right) p=q a a^{\dagger} a p=q a p . \\
& a^{\#}(q a p) a^{\#}=(p+q-\mathbb{1})^{-1} a^{\dagger}\left((p+q-\mathbb{1})^{-1} q a\right) p(p+q-\mathbb{1})^{-1} a^{\dagger}(p+q-\mathbb{1})^{-1} \\
&=(p+q-\mathbb{1})^{-1} a^{\dagger}\left(a p(p+q-\mathbb{1})^{-1}\right) a^{\dagger}(p+q-\mathbb{1})^{-1} \\
&=(p+q-\mathbb{1})^{-1} a^{\dagger} a a^{\dagger}(p+q-\mathbb{1})^{-1} \\
&=(p+q-\mathbb{1})^{-1} a^{\dagger}(p+q-\mathbb{1})^{-1}=a^{\#} . \\
& a^{\#}(q a p)=(p+q-\mathbb{1})^{-1} a^{\dagger}\left((p+q-\mathbb{1})^{-1} q a\right) p \\
&=(p+q-\mathbb{1})^{-1} a^{\dagger} a p=(p+q-\mathbb{1})^{-1} q a a^{\dagger}=a a^{\dagger} \text { is self-adjoint. } \\
&(q a p) a^{\#}=q\left(a p(p+q-\mathbb{1})^{-1}\right) a^{\dagger}(p+q-\mathbb{1})^{-1} \\
&=q a a^{\dagger}(p+q-\mathbb{1})^{-1}=a^{\dagger} a p(p+q-\mathbb{1})^{-1}=a^{\dagger} a \text { is self-adjoint. }
\end{aligned}
$$


Thus, qap $\in \mathcal{A}^{\dagger}$ and $(q a p)^{\dagger}=a^{\#}$. Hence qap $=\left((q a p)^{\dagger}\right)^{\dagger}=\left(a^{\#}\right)^{\dagger}$.

Observe that the proof of former result distils that if $a \in \mathcal{A}^{\#}$, then

$$
a^{\#}\left(a^{\#}\right)^{\dagger}=a a^{\dagger} \quad \text { and } \quad\left(a^{\#}\right)^{\dagger} a^{\#}=a^{\dagger} a .
$$

The following elementary fact will be also useful. Let $\mathcal{A}$ be a Banach algebra with identity and let $\left\{b_{n}\right\}_{n=1}^{\infty} \subset \mathcal{A}$ be a sequence with limit $b \in \mathcal{A}$. If $b_{n} \in \mathcal{A}$ are invertible, and if $\sup \left\|b_{n}^{-1}\right\|<\infty$, then $b$ is invertible and $b_{n}^{-1} \rightarrow b^{-1}$.

To prove the main result, we need to remark that for $a_{n} \in \mathcal{A}^{\dagger}$ such that $a_{n} \rightarrow a$, the following holds:

$a \in \mathcal{A}^{\dagger}, a_{n}^{\dagger} \rightarrow a^{\dagger} \Leftrightarrow \sup _{n}\left\|a_{n}^{\dagger}\right\|<\infty \Leftrightarrow a \in \mathcal{A}^{\dagger}, a_{n}^{\dagger} a_{n} \rightarrow a^{\dagger} a \Leftrightarrow a \in \mathcal{A}^{\dagger}, a_{n} a_{n}^{\dagger} \rightarrow a a^{\dagger}$.

Evidently, the only difference between (2.4) and the well-known necessary and sufficient conditions for the continuity of the Moore-Penrose inverse which are given in ( [17, Theorem 2.2], [8, Theorem 6], [19, Theorem 2.2]), is the implication

$$
\sup _{n}\left\|a_{n}^{\dagger}\right\|<\infty \Rightarrow a \in \mathcal{A}^{\dagger} \text {. }
$$

It follows by the following: Since

$a_{n}^{\dagger}-a_{m}^{\dagger}=-a_{n}^{\dagger}\left(a_{n}-a_{m}\right) a_{m}^{\dagger}+a_{n}^{\dagger} a_{n}^{* \dagger}\left(a_{n}^{*}-a_{m}^{*}\right)\left(\mathbb{1}-a_{m} a_{m}^{\dagger}\right)+\left(\mathbb{1}-a_{n}^{\dagger} a_{n}\right)\left(a_{n}^{*}-a_{m}^{*}\right) a_{m}^{* \dagger} a_{m}^{\dagger}$, we get that $a_{n}^{\dagger}$ is a Cauchy sequence, and thus convergent to an element $b \in \mathcal{A}$.

Letting $n \rightarrow \infty$ in $a_{n} a_{n}^{\dagger} a_{n}=a_{n}$ we get $a b a=a$. Thus, $a$ is regular, hence Moore-Penrose invertible.

In the following theorem we present some necessary and sufficient conditions for the continuity of the group inverse. As we mentioned before that problem was considered in [12]. Here, we don't have an additional assumption that a limit $a$ is group invertible.

Theorem 2.7. Let $\mathcal{A}$ be a $C^{*}$-algebra and let $\left\{a_{n}\right\}_{n=1}^{\infty}$ be a sequence of nonzero elements from $\mathcal{A}^{\#}$ that converges to $a \in \mathcal{A}$. The following statements are equivalent:

(i) $a \in \mathcal{A}^{\#}$ and $a_{n}^{\#} \rightarrow a^{\#}$.

(ii) $\left\{a_{n}^{\#}\right\}_{n=1}^{\infty}$ is a bounded sequence.

(iii) $a \in \mathcal{A}^{\dagger}, a_{n}^{\dagger} \rightarrow a^{\dagger}$ and there exist $\psi \in[0, \pi / 2)$ and $n_{0} \in \mathbb{N}$ such that $\psi\left(a_{n} a_{n}^{\dagger}, a_{n}^{\dagger} a_{n}\right) \leq \psi$ for all $n \geq n_{0}$.

Proof. Let us denote $\pi_{n}=a_{n}^{\#} a_{n}, p_{n}=a_{n} a_{n}^{\dagger}$, and $q_{n}=a_{n}^{\dagger} a_{n}$.

(i) $\Rightarrow$ (ii) is evident.

(ii) $\Rightarrow$ (iii): From (2.1) and Corollary 2.1 (ii) of [2] we have

$$
\left\|a_{n}^{\dagger}\right\|=\left\|\left(p_{n}+q_{n}-\mathbb{1}\right) a_{n}^{\#}\left(p_{n}+q_{n}-\mathbb{1}\right)\right\| \leq\left\|p_{n}+q_{n}-\mathbb{1}\right\|^{2}\left\|a_{n}^{\#}\right\| \leq\left\|a_{n}^{\#}\right\| .
$$

Hence $\sup \left\|a_{n}^{\dagger}\right\|<\infty$, and using (2.4), we obtain $a \in \mathcal{A}^{\dagger}$ and $a_{n}^{\dagger} \rightarrow a^{\dagger}$. By Theorem 2.5 we have

$$
\frac{1}{\cos \psi\left(p_{n}, q_{n}\right)}=\left\|\pi_{n}\right\| \leq\left\|a_{n}\right\|\left\|a_{n}^{\#}\right\|
$$


Since $\left\{a_{n}\right\}_{n=1}^{\infty}$ and $\left\{a_{n}^{\#}\right\}_{n=1}^{\infty}$ are convergent, there exists $\delta>0$ such that $\delta \leq$ $\cos \psi\left(p_{n}, q_{n}\right)$ for any $n \in \mathbb{N}$. By setting $\psi=\arccos \delta$ allows us to get (iii).

(iii) $\Rightarrow$ (i): By Theorem 2.6, we have that $\left\{\left(a_{n}^{\#}\right)^{\dagger}\right\}_{n=1}^{\infty}$ is a convergent sequence. For any $n \in \mathbb{N}$, let $b_{n}=\left(a_{n}^{\#}\right)^{\dagger}$. By [2, Cor. 2.1] and Theorem 2.4 we get

$$
\left\|b_{n}^{\dagger}\right\|=\left\|a_{n}^{\#}\right\|=\left\|\left(p_{n}+q_{n}-\mathbb{1}\right)^{-1} a_{n}^{\dagger}\left(p_{n}+q_{n}-\mathbb{1}\right)^{-1}\right\| \leq \frac{\left\|a_{n}^{\dagger}\right\|}{\cos ^{2} \psi\left(p_{n}, q_{n}\right)} \leq \frac{\left\|a_{n}^{\dagger}\right\|}{\cos ^{2} \psi}
$$

Since $\left\{a_{n}^{\dagger}\right\}_{n=1}^{\infty}$ is bounded, by (2.5) we have that $\left\{b_{n}^{\dagger}\right\}_{n=1}^{\infty}$ is bounded. By (2.4), the sequence $\left\{b_{n}^{\dagger}\right\}_{n=1}^{\infty}$ converges to some element, say $r \in \mathcal{A}$. In other words, $\lim _{n \rightarrow \infty} a_{n}^{\#}=r$. Now, by letting $n \rightarrow \infty$ in the equalities

$$
a_{n}=a_{n} a_{n}^{\#} a_{n}, \quad a_{n}^{\#}=a_{n}^{\#} a_{n} a_{n}^{\#}, \quad a_{n} a_{n}^{\#}=a_{n}^{\#} a_{n},
$$

we obtain, respectively, $a=a r a, r=r a r$, and $a r=r a$, from which we get that $a$ is group invertible and $a^{\#}=r=\lim _{n \rightarrow \infty} a_{n}^{\#}$.

Next corollary shows how the speed of the convergence of $a_{n}^{\#}$ and $a_{n} a_{n}^{\#}$ depends on three items: (a) $\left\|a_{n}^{\dagger}\right\|$ and $\left\|a^{\dagger}\right\|$; (b) $\psi\left(a_{n} a_{n}^{\dagger}, a_{n}^{\dagger} a_{n}\right)$ and $\psi\left(a a^{\dagger}, a^{\dagger} a\right)$; (c) $\left\|a-a_{n}\right\|$. Also it shows that the closer is $\psi\left(a_{n} a_{n}^{\dagger}, a_{n}^{\dagger} a_{n}\right)$ to $\pi / 2$, the worst is the convergence $a_{n}^{\#}$ and $a_{n} a_{n}^{\#}$.

Let $a_{n}^{\pi}$ and $a^{\pi}$ be the spectral idempotents of $a_{n}$ and $a$ defined by $a_{n}^{\pi}=\mathbb{1}-a_{n} a_{n}^{\#}$ and $a^{\pi}=\mathbb{1}-a a^{\#}$, respectively.

Corollary 2.8. Let $\mathcal{A}$ be a $C^{*}$-algebra. Let $\left\{a_{n}\right\}_{n=1}^{\infty}$ be a sequence of nonzero elements in $\mathcal{A}^{\#}$ that converges to a $\in \mathcal{A}^{\dagger}$. If exists $\psi<\pi / 2$ such that $\psi\left(a_{n} a_{n}^{\dagger}, a_{n}^{\dagger} a_{n}\right) \leq$ $\psi$ for any $n \in \mathbb{N}$ and $\psi\left(a a^{\dagger}, a^{\dagger} a\right) \leq \psi$, then

$$
\left\|a_{n}^{\#}-a^{\#}\right\| \leq\left[\frac{\left\|a_{n}^{\dagger}\right\|\left\|a^{\dagger}\right\|}{\cos ^{2} \psi}+\frac{\left\|a_{n}^{\dagger}\right\|^{2}+\left\|a^{\dagger}\right\|^{2}}{\cos ^{3} \psi}\right]\left\|a_{n}-a\right\|
$$

and

$$
\left\|a_{n} a_{n}^{\#}-a a^{\#}\right\| \leq\left[\frac{\left\|a_{n}^{\dagger}\right\|+\left\|a^{\dagger}\right\|}{\cos ^{2} \psi}\right]\left\|a_{n}-a\right\| .
$$

Proof. Let $p=a a^{\dagger}, q=a^{\dagger} a, p_{n}=a_{n} a_{n}^{\dagger}$, and $q_{n}=a_{n}^{\dagger} a_{n}$. By [13, Prop. 4.2], [2, Cor. 2.1], and Theorem 2.4, one gets

$\left\|a^{\pi}\right\|=\left\|\mathbb{1}-a a^{\#}\right\|=\left\|a a^{\#}+\left(a a^{\#}\right)^{*}-\mathbb{1}\right\|=\left\|(p+q-\mathbb{1})^{-1}\right\|=\frac{1}{\cos \psi(p, q)} \leq \frac{1}{\cos \psi}$.

The proof of Theorem 2.7 (see (2.5)) enables us to get

$$
\left\|a^{\#}\right\| \leq \frac{\left\|a^{\dagger}\right\|}{\cos ^{2} \psi(p, q)} \leq \frac{\left\|a^{\dagger}\right\|}{\cos ^{2} \psi}
$$


Similar expressions as in (2.8), (2.9) can be obtained by changing $a$ by $a_{n}$. Observe that

$$
\begin{aligned}
a_{n}^{\#}-a^{\#} & =a_{n}^{\#}-a^{\#}+a_{n}^{\#}\left(a_{n}-a\right) a^{\#}-a_{n}^{\#}\left(a_{n}-a\right) a^{\#} \\
& =a_{n}^{\#}\left(\mathbb{1}-a^{\#} a\right)-\left(\mathbb{1}-a_{n}^{\#} a_{n}\right) a^{\#}-a_{n}^{\#}\left(a_{n}-a\right) a^{\#} \\
& =\left(a_{n}^{\#}\right)^{2} a_{n} a^{\pi}-a_{n}^{\pi} a\left(a^{\#}\right)^{2}-a_{n}^{\#}\left(a_{n}-a\right) a^{\#} \\
& =\left(a_{n}^{\#}\right)^{2}\left(a_{n}-a\right) a^{\pi}-a_{n}^{\pi}\left(a-a_{n}\right)\left(a^{\#}\right)^{2}-a_{n}^{\#}\left(a_{n}-a\right) a^{\#},
\end{aligned}
$$

hence we have

$$
\left\|a_{n}^{\#}-a^{\#}\right\| \leq\left[\left\|a_{n}^{\#}\right\|\left\|a^{\#}\right\|+\left\|a_{n}^{\#}\right\|^{2}\left\|a^{\pi}\right\|+\left\|a_{n}^{\pi}\right\|\left\|a^{\#}\right\|^{2}\right]\left\|a_{n}-a\right\| .
$$

Now (2.6) follows from (2.8), (2.9), and (2.10). The identity $a_{n} a_{n}^{\#}-a a^{\#}=$ $a_{n}^{\#}\left(a_{n}-a\right) a^{\pi}+a_{n}^{\pi}\left(a_{n}-a\right) a^{\#}$ is trivial to check. Hence

$$
\left\|a_{n} a_{n}^{\#}-a a^{\#}\right\| \leq\left[\left\|a_{n}^{\#}\right\|\left\|a^{\pi}\right\|+\left\|a_{n}^{\pi}\right\|\left\|a^{\#}\right\|\right]\left\|a_{n}-a\right\| .
$$

Now (2.7) follows again from (2.8) and (2.9).

Acknowledgement. We would like thank the referee for his/her valuable comments.

The first author was supported by the Universidad Politécnica de Valencia (PAID-06-12). The second author is supported by Grant No. 174007 of the Ministry of Science, Technology and Development, Republic of Serbia. The third author is supported by the Guangxi Natural Science Foundation 2013GXNSFAA019008, the key project of Education Department of Guangxi 201202ZD031 Project and by national science foundation of China 11361009.

\section{REFERENCES}

1. J. Benítez and V. Rakočević, Invertibility of the commutator of an element in a $C^{*}$-algebra and its Moore-Penrose inverse, Studia Math. 200 (2010), 163-174.

2. J. Benítez and D. Cvetković-Ilić, On the elements $a a^{\dagger}$ and $a^{\dagger} a$ in a ring, Appl. Math. Comput. 222 (2013), 478-489.

3. D. Buckholtz, Hilbert space idempotents and involutions, Proc. Amer. Math. Soc. 128 (2000), 1415-1418.

4. D. Buckholtz, Inverting the difference of Hilbert space projections, Amer. Math. Monthly 104 (1997), 60-61.

5. S.L. Campbell and C.D. Meyer, Jr., Continuity Properties of the Drazin Pseudoinverse, Linear Algebra Appl. 10 (1975), 77-83.

6. M.P. Drazin, Pseudoinverse in associative rings and semigroups, Amer. Math. Monthly 65 (1958), 506-514.

7. A. Galántai, Subspaces, angles and pairs of orthogonal projections, Linear Multilinear Algebra 56 (2008), 227-260.

8. R. Harte and M. Mbekhta, On generalized inverses in $C^{*}$-algebras II, Studia Math. 106 (1993), 129-138.

9. Q. Huang, J. Yu and L. Zhu, Some new perturbation results for generalized inverses of closed linear operators in Banach spaces, Banach J. Math. Anal. 6 (2012), 58-68.

10. I.C.F. Ipsen and C.D. Meyer, The angle between complementary subspaces, Amer. Math. Monthly 102 (1995), 904-911.

11. S. Izumino, Convergence of generalized inverses and spline projectors, J. Approx. Theory 38 (1983), 269-278. 
12. J.J. Koliha and V. Rakočević, Continuity of the Drazin inverse II, Studia Math. 131 (1998), 167-177.

13. J.J. Koliha, Range projections of idempotents in $C^{*}$-algebras, Demonstratio Math. 34 (2001), 91-103.

14. J.J. Koliha and V. Rakočević, On the norm of idempotents in $C^{*}$-algebras, Rocky Mountain J. Math., 34 (2004), 685-697.

15. V.E. Ljance, Certain properties of idempotent operators (Russian) Tr. Inst. Prikl. Mat. Mekh. 1 (1958), 16-22.

16. S. Maeda, On the distance between two projections in $C^{*}$-algebras, Math. Japon. 22 (1977), $61-65$.

17. M. Mbekhta, Conorme et inverse généralisé dans les $C^{*}$-algèbres, Canad. Math. Bull. 35 (1992), 515-522.

18. V. Rakočević, Continuity of the Drazin inverse, J. Operator Theory, 41 (1999), 55-68.

19. V. Rakočević, On the continuity of the Moore-Penrose inverse in $C^{*}$-algebras, Math. Montisnigri 2 (1993), $89-92$.

20. G.W. Stewart, On the continuity of the generalized inverse, SIAM J. Appl. Math. 17 (1969), $33-45$.

1 Departamento de Matemática Aplicada, Instituto de Matemática MultidisCiplinar, Universidad Politécnica de Valencia, Camino de Vera s/n, 46022, VaLENCIA, SPAin.

E-mail address: jbenitez@mat.upv.es

2 University of Niš, Department of Mathematics, Faculty of Sciences and Mathematics, 18000 Niš, Serbia.

E-mail address: gagamaka@ptt.rs

${ }^{3}$ College of Mathematics and Computer Science, Guangxi University for Nationalities, Nanning 530006, China.

E-mail address: xiaojiliu72@126.com 